\section{Kollektive Verantwortung und Armut}

Die philosophische Literatur zu kollektiver Verantwortung und Armut bezieht sich fast ausschließlich auf globale Armut. Daher wird der Fokus dieses Betrags ebenso auf unserer kollektiven Verantwortung für globale Armut liegen, wobei es natürlich vorrangig um konzeptionelle Frage geht.

Die Frage nach der Verantwortung für globale Armut lässt sich auf mindestens zwei Weisen stellen - als Frage nach der (retrospektiven) Verantwortung für das Auftreten dieses Problems oder als Frage nach der (prospektiven) Verantwortung für dessen Behebung. Beide Fragen sind insofern miteinander verbunden als dass Verantwortung für das Auftreten eines Problems normalerweise Verantwortung für dessen Behebung mit sich zieht. Da dies jedoch nicht zwingend der Fall ist, und zudem prospektive Verantwortung für die Behebung eines moralischen Übels auch ohne jedwede retrospektive Verantwortung aufkommen kann, lassen sich beide Fragen auch getrennt voneinander behandeln. Dieses Kapitel wird sich vor allem auf die zweite Frage konzentrieren: Inwiefern sollte die Verantwortung, Armut zu bekämpfen, als kollektive Verantwortung verstanden werden? Insofern wird im Folgenden von Verantwortung zumeist im prospektiven Sinn gesprochen. Mitunter wird dafür auch der Begriff der moralischen Pflicht verwendet.

Hier wird nicht dafür argumentiert, dass globale Armut ein moralisches Problem und dessen Behebung geboten ist. Es wird davon ausgegangen, dass über diese Frage weitgehende Einigkeit herrscht. Es wird ebenso wenig diskutiert, wie weitreichend unsere Pflichten der Armutsbekämpfung sind - dies kann nur im Rahmen einer substantiellen Moraltheorie getan werden, und die Ausführungen hier sind neutral in Bezug auf eine solche Theorie.

Zahlreiche Autor*innen plädieren dafür, unsere (prospektive) Verantwortung für globale Armut als kollektive zu bezeichnen (Ashford 2013; Calder 2010; Schwenkenbecher 2017; 2020; Wringe 2005; 2010; 2014; Young 2004; 2011; Cullity 2004; Murphy 2000; Schlothfeldt 2009).

Im Folgenden wird zunächst erläutert, aus welchen Gründen Philosophinnen die Idee der kollektiven Verantwortung für Armutsbekämpfung verteidigen, bzw. was diese Idee motiviert. Im Zuge dessen werden die Probleme des kollektiven Verantwortungsbegriffs diskutiert. Im Anschluss daran wird dargelegt, in wel- chem Sinne Verantwortung und Hilfspflichten als kollektiv verstanden werden können.

\subsection{Warum kollektive Verantwortung?}

Es gibt zweierlei Gründe, die Philosoph*innen anführen, um die Idee der kollektiven Verantwortung für Weltarmut zu motivieren: Erstens, die Tatsache, dass Weltarmut kollektiv verursacht wird, dass also unsere retrospektive Verantwortung einen gemeinsame ist, und zweitens die Einsicht, dass dieses Problem nur von einer Vielzahl von Akteurinnen gemeinsam behoben werden kann, im Sinne einer prospektiven Verantwortung.

In welchem Sinne kann die Verursachung von globaler Armut als ein kollektiv herbeigeführtes Problem verstanden werden? Autor ${ }^{\star}$ innen sind sich einig, dass die Verursachung von globaler Armut nicht das Resultat gemeinsamer Handlungen im engeren Sinn ist. Mit anderen Worten, es ist nicht der Fall, dass die kausal an der Erzeugung von Armut beteiligten Akteur*innen diesbezüglich gemeinsame Handlungsabsichten teilen und gezielt auf dieses Ergebnis hinarbeiten bzw. hingearbeitet haben.

Das Problem lässt sich besser als ein Fall struktureller Ungerechtigkeit verstehen. Iris Marion Young und Elizabeth Ashford, zum Beispiel, argumentieren, dass normale Alltagshandlungen $\mathrm{zu}$ struktureller Ungerechtigkeit, zum Beispiel in Form von globaler Armut, wesentlich beitragen, wenn diese Alltagshandlungen in ein System ungerechter sozialer Institutionen einbettet sind (Young 2011, Ashford 2013). Corwin Aragon und Alison Jaggar schreiben "structural injustice is an emergent property of social practices«, ein Resultat von »typically unplanned and often unforeseen interaction of a variety of system factors « (Aragon/Jaggar 2018, 442).

Während Thomas Pogge darauf verweist, dass unsere Pflichten der Armutsbekämpfung in der Verletzung einer negativen Pflicht, andere nicht zu schädigen, begründet sind (Pogge 2002), plädiert Judith Lichtenberg dafür, derlei Schädigungen als ınew harms` zu verstehen (Lichtenberg 2010). Solch ein neuer Schadensbegriff soll helfen, die Komplexität von kollektiv herbeigeführten Schädigungen besser zu erfassen:

»We see-or, in many cases, others inform us in no uncertain terms-that our most humdrum activities may harm people in myriad ways we have never thought about before. And because these activities are seamlessly woven into our normal routines, ceasing to engage in 
these $>$ New Harms $<$ is not at all easy-not simply a matter of refraining from things we never would have dreamed of doing in the first place« (ebd., 558).

Das Zitat von Lichtenberg greift bereits meinen nächsten Gedanken auf - das Problem der Behebung von kollektiv herbeigeführten moralischen Übeln. Selbst wenn wir davon ausgehen, dass die ursächliche Verantwortung für Armut kollektiver Natur, d. h., eine gemeinsame, ist, und scheinbar harmlose Einzelhandlungen kumulativ wesentlich dazu beitragen, dann ist damit noch nicht zugleich eine Lösung für dieses Problem gefunden. Wie Lichtenberg feststellt, ist es nicht unbedingt der Fall, dass wir Armut durch das bloße Unterlassen besagter Einzelhandlungen ohne Weiteres beenden könnten. Ohne die Bedeutsamkeit der Frage nach der retrospektiven Verantwortung für Armut und andere Formen struktureller Ungerechtigkeit anzweifeln zu wollen, wird hier vor allem der Frage nach dem kollektiven Charakter unserer prospektiven Verantwortung fokussiert.

Der hauptsächliche Grund, für eine kollektive Art der prospektiven Verantwortung für Weltarmut $\mathrm{zu}$ plädieren, ist die Notwendigkeit kollektiven Handelns für die Bekämpfung derselben. Man kann hier von sogenannter kollektiver Notwendigkeit sprechen. Kollektive Notwendigkeit besteht dann, wenn in der entsprechenden Situation »ein moralisch gewünschter Zustand nur von mehreren Akteuren gemeinsam hergestellt werden kann. Kein Akteur ist allein in der Lage, den gewünschten Zustand herbeizuführen « (Schwenkenbecher 2017, 126; vgl. auch Lawford-Smith 2012).

Um zu illustrieren, inwiefern kollektive Notwendigkeit kollektive Pflichten (im Sinne von prospektiver kollektiver Verantwortung) erzeugt, verweisen Autor*innen üblicherweise auf überschaubare Beispielsfälle kollektiven Handelns. Garrett Cullity, zum Beispiel, beschreibt den folgenden Fall gemeinsamen Handlungsbedarfs, mit dem Ziel, damit eine gemeinsame globale Hilfspflicht zu illustrieren:

"If someone is drowning in front of you and me, and can be rescued only by using a winch mechanism that requires two people to operate, then it is obvious that we are morally required to help him, even if neither of us could do so single-handedly“ (Cullity 2004, 61).

Ähnliche Beispielsfälle werden auch von anderen $\mathrm{Au}-$ tor*innen zur Begründung kollektiver Pflichten herangezogen (Schwenkenbecher 2013; 2017; Wringe 2016). Die grundlegende Idee ist wie folgt: da in einem solchen Notfall die potentiellen Retter*innen nur gemein- sam ein Menschenleben retten können, kann die Pflicht, dies zu tun, nur eine gemeinsame sein. Denn wir können nicht zu etwas verpflichtet sein, das wir (allein) nicht ausführen können. Bzw. mit anderen Worten, wenn ich grundsätzlich nicht in der Lage bin, eine bestimmte Handlung aus- bzw. ein bestimmtes Resultat herbeizuführen, dann kann ich auch nicht eine moralische Pflicht haben, dies zu tun. Dieses Prinzip wird im Englischen meist mit ought implies can ausgedrückt. Die o. g. Autor*innen gehen entweder implizit oder explizit davon aus, dass eine gemeinsame Pflicht dann bestehen kann, wenn gemeinsame Handlungsfähigkeit herrscht. In einem zweiten Schritt gehen viele Autor ${ }^{\star}$ innen davon aus, dass umfassende, globale Probleme wie Weltarmut und Klimawandel, eine diesen Beispielsfällen ausreichend ähnliche Struktur aufweisen und somit parallele Schlussfolgerungen bezüglich unserer gemeinsamen moralischen Verantwortung gerechtfertigt sind.

Leider wird dieser Schritt in der Literatur so gut wie nie begründet, was hier nicht ausführlich diskutiert werden kann (vgl. dazu Schwenkenbecher 2017; 2020). Tatsache ist, dass die Hintergrundbedingungen für sehr überschaubare Rettungsfallszenarien mit einer Handvoll von Beteiligten einerseits und die für globale Probleme struktureller Ungerechtigkeit mit einer unüberschaubaren Zahl und Vielfalt von Akteur*innen andererseits komplett verschieden sind. Diese Unterschiede sind meines Erachtens normativ relevant, d. h. sie haben eine Auswirkung auf die Art der gemeinsamen Pflichten, die Akteur*innen zukommen können.

In idealisierten Rettungsfällen herrscht nicht nur sogenannte moralische Dringlichkeit - das heißt, dass nur durch rasches Handeln ein immanenter, schwerwiegender Verlust, wie z. B. ein Menschenleben, verhindert werden kann. Es besteht epistemische und moralische Eindeutigkeit, was die zur Handlung notwendigen Akteur*innen und deren jeweilige Handlungsstrategien betrifft: es ist jeweils klar, wer wie handeln muss, um das Problem ein für alle Mal zu beheben. Es handelt sich stets um einmalig auftretende Ausnahmefälle, nicht um anhaltende Probleme. Die Strategien zur Lösung dieser Probleme sind dabei wenig komplex. Sie sind für die Akteur*innen, welche sich damit konfrontiert sehen, nicht nur offensichtlich, sondern auch einfach auszuführen. Die Akteur*innen und potentiellen Helfer ${ }^{\star}$ innen sind epistemisch dem Problem ausgeliefert: sie sind direkt und unausweichlich damit konfrontiert und es bestehen in der Abstraktheit, mit der die Fälle von den verschiedenen Autor ${ }^{\star}$ innen präsentiert 
werden, keine kognitive Konkurrenz. Das Problem und dessen Lösung, sowie die Notwendigkeit dringenden Eingreifens, werden in dieser Art von Fällen von moralischen Durchschnittsmenschen direkt und intuitiv erfasst.

All dies ist mit komplexeren, anhaltenden Problemen wie globaler Armut nicht der Fall. Deren Lösung verlangt kontinuierliches, koordiniertes Engagement von Gruppenakteur*innen, wie Staaten, (über)staatlichen Organisationen, Firmen und Hilfsorganisationen, sowie Einzelakteur*innen - entweder in führenden oder unterstützenden Rollen. Lösungsstrategien sind für Einzelakteur*innen nicht unmittelbar erfassbar - sie erfordern sorgfältige Recherche und Expertise - und schon gar nicht umsetzbar - der Beitrag Einzelner ist meist nur ein kleiner Teil eines komplexen Puzzles.

All dies bedeutet meines Erachtens nicht, dass keine gemeinsame Verantwortung zur Behebung derart komplexer moralischer Übel besteht, bzw. dass Einzelakteur*innen an dieser Verantwortung nicht teilhaben. Allerdings sollte es nahelegen, dass die Art der gemeinsamen Verantwortung für komplexe und weitreichende Probleme struktureller Natur, wie globale Armut oder Klimawandel, nicht dieselbe sein kann, wie die gemeinsame Verantwortung, die Einzelakteur*innen in spontanen Notfällen wie dem oben beschriebenen Rettungsfall zukommt. Hier wird daher weiter unten zwischen starker und schwacher kollektiver Verantwortung unterschieden.

Was kann also sinnvollerweise unter einer gemeinsamen bzw. kollektiven Verantwortung für die Bekämpfung von globaler Armut verstanden werden?

\subsection{Was ist mit kollektiver Verantwortung gemeint?}

Wie bereits gesagt, ist von kollektiver Verantwortung im prospektiven Sinn dann die Rede, wenn zwei oder mehr Akteur*innen gemeinsam eine Pflicht haben, eine Handlung auszuführen bzw. ein moralisch wertvolles Ergebnis herbeizuführen. Aber in welchem Sinn ist eine solche Verantwortung >kollektiv`? Grob gesehen, kann man in der Literatur drei verschiedene Konzeptionen von kollektiver Verantwortung vorfinden (vgl. auch Schwenkenbecher 2020):

1. Kollektive Verantwortung als Verantwortung einer Gruppe. Dies kann zweierlei bedeuten:

a) Die Verantwortung eines/einer Gruppenakteur ${ }^{*}$ in: Hier wird davon ausgegangen, dass Gruppen von moralischen Akteur*innen selbst moralische Akteur`innen sein können, denen Verantwortung bzw. Pflichten zugeschrieben werden können und deren Pflichten nicht auf die Summe der Pflichten der Gruppenmitglieder reduzierbar sind. Ein Argument für den moralischen Status von sogenannten konstituierten Gruppenakteur ${ }^{\star}$ innen, wie zum Beispiel Firmen und Staaten, als moralische Akteur ${ }^{\star}$ innen findet sich unter anderem in Christian Lists und Philip Pettits Group Agency (2011).

Obgleich man ebenfalls davon ausgehen mag, dass derartige Gruppen moralische Akteur*innen sein können, bedeutet dies nicht unbedingt, dass diese Art der kollektiven Verantwortung besonders einschlägig für das Problem der (globalen) Armut ist. Immerhin müsste hier eine Vielzahl von Gruppen- und Einzelakteur ${ }^{\star}$ innen zusammen an einer Lösung arbeiten. Diese Akteur*innen bilden zusammen keine ${ }^{*} n$ $\left(\right.$ neue $\left.^{\star} n\right)$ Gruppenakteur ${ }^{\star}$ in im engeren Sinne.

b) Die Verantwortung einer Gruppe, die kein ${ }^{*}$ Akteur ${ }^{*}$ in im strengen Sinne ist. Diese vor allem von Bill Wringe verteidigte Ansicht besagt, dass Gruppen, die keine Organisationsstruktur im o. g. Sinn aufweisen, dennoch moralische Pflichten haben können. Das Problem dieser Interpretation ist, dass sie das sogenannte Akteursprinzip verletzt, demgemäß nur moralische Akteur*innen Pflichtenträger*innen sein können. Bill Wringe argumentiert, dass das Akteursprinzip hinfällig ist (Wringe 2010).

2. Als gemeinsame Pflichten von zwei oder mehr Akteur*innen: >Gemeinsam verpflichtet sein wird hier als plurales Prädikat verstanden, welches Sets von Akteur*innen zugeschrieben werden kann (ein anderes Beispiel für ein plurales Prädikat ist raneinander vorbeigehen`) (Schwenkenbecher 2013; 2019; Pinkert 2014). In diesem Sinne ist ebenfalls Gunnar Björnssons Begriff der 'geteilten Verantwortung zu verstehen, demgemäß zwei oder mehrere Akteure dann eine Pflicht teilen, wenn eine angemessene Reaktion auf moralische Gründe und die richtige moralische Motivation den Akteure eine gemeinsame Handlung nahelegen würde (Björnsson 2014).

3. Als Pflichten individueller Akteur*innen, die sich auf ein gemeinsames Ziel beziehen: Jeder der Einzelakteur ${ }^{*}$ innen hat eine Pflicht zur einer kollektiven Handlung beizutragen (Collins 2013, Aas 2015). Das Problem dieser Interpretation gemeinsamer Pflichten ist, dass letztendlich kein ${ }^{\star}$ Akteur*in und kein Set von Akteur*innen die Pflicht hat, die erforderliche Handlung auszuführen bzw. das gewünschte Ergebnis zu erzielen. Diese Pflicht kann nicht zugeschrieben 
werden, die Einzelakteur*innen haben lediglich Beitrags- und Ermöglichungspflichten.

Es würde hier zu weit führen, diese verschiedenen Interpretationen im Detail zu diskutieren (vgl. dazu Schwenkenbecher 2018). Die Nachteile jeder Interpretation wurden jeweils angedeutet. Es lohnt jedoch, festzuhalten, dass die oben beschriebenen Interpretationen sich nicht gegenseitig ausschließen müssen. Es ist höchstwahrscheinlich vorteilhaft, dafür offen zu sein, dass Verantwortung und moralische Pflichten auf unterschiedliche Weise gemeinsamer bzw. kollektiver Natur sein können. Der Vollständigkeit halber, sollte erwähnt werden, dass man in Bezug auf die oben beschriebenen spontanen Rettungsfälle die zweite Interpretation gemeinsamer Pflichten als plausibler betrachten kann, verglichen mit den Alternativen. Es ist jedoch fragwürdig, ob sich diese in Bezug auf überschaubare Rettungsfälle entwickelte Konzeption ohne Weiteres auf großangelegte, komplexe Problem wie globale Armut übertragen lässt (Schwenkenbecher 2013; 2017; 2020).

\subsection{Kollektive Verantwortung und (globale) Armut}

In welchem Sinne könnte also unsere prospektive Verantwortung zur Verminderung (und letztlich Behebung) globaler Armut kollektiver Natur sein? Damit könnte einerseits die gemeinsame Verantwortung all derjenigen Akteur*innen gemeint sein, die zur Behebung des Problems beitragen können. Dies würde, wie bereits erwähnt, sowohl sogenannte konstituierte Gruppenakteur*innen wie Staaten und deren Institutionen, aber auch überstaatliche Gruppen, Firmen, Nichtregierungs-organisationen und Einzelpersonen umfassen. Allerdings wird der Begriff der kollektiven Verantwortung für Weltarmut in der philosophischen Literatur fast ausschließlich im Hinblick auf eine gemeinsame Pflicht von nicht-organisierten Einzelpersonen gebraucht. Sozusagen als eine gemeinsame Pflicht aller Weltbürger*innen, oder auch nur der Einwohner ${ }^{*}$ innen der ersten Welt.

Was erhoffen sich Autorinnen von einer /Kollektivierung ¿ des Verantwortungs- bzw. Pflichtbegriffs in diesem Zusammenhang? Inwiefern sollte die Rede von kollektiver Verantwortung dabei helfen, unsere moralischen Verwicklungen in Probleme dieser Dimension besser zu verstehen bzw. uns aufzeigen, wie wir über deren Lösung denken sollten?

Offenbar verfolgen Autor ${ }^{\star}$ innen, die einen solchen kollektiven Verantwortungsbegriff vertreten, üblicherweise mindestens eine der zwei folgenden Strategien.

1. Normativer Vorrang des Kollektiven: Wie bereits erwähnt, liegt ein Grund, unsere Verantwortung (bzw. Pflichten) in Bezug auf weitreichende kollektive Handlungsprobleme als gemeinsame zu verstehen darin, dass nur gemeinsames Handeln diese Probleme beheben kann und dass Einzelakteur*innen (und Einzelhandlungen) für sich genommen dies nicht vermögen. Es ist die gemeinsame Fähigkeit mehrerer Akteur*innen, diese Probleme zu lösen, die eine kollektive Pflicht begründet.

Zudem hilft die Annahme, dass solche Pflichten kollektiv sind, über zwei bekannte moraltheoretische Probleme hinweg, nämlich erstens die Tatsache, dass in vielen Fällen die Beiträge Einzelner (sowie auch die Beitragsunterlassungen Einzelner) keinen messbaren Einfluss auf das Endresultat haben und zweitens dass sie für dieses Endresultat nicht zwingend notwendig sind.

Nehmen wir Klimawandel als Beispiel zur Illustration des ersten Problems. Es ist eine Tatsache, dass mein $\mathrm{CO}_{2}$-Fußabdruck - für sich genommen - keinerlei messbaren Einfluss auf die Erderwärmung hat, ebenso wenig hätte dies die Verminderung des von mir verursachten $\mathrm{CO}_{2}$ Ausstoßes. Dies hat sowohl mit der Komplexität des Problems, als auch mit dessen Ausmaßen zu tun. Die meisten der uns unmittelbar zur Verfügung stehenden Handlungen machen - einzeln genommen - keinen spür- oder messbaren Unterschied. Wenn unsere moralischen Pflichten zur Behebung von Übeln an unsere Fähigkeit geknüpft sind, als Einzelakteur ${ }^{*}$ innen direkten Einfluss auf die Behebung solcher Probleme auszuüben, d. h. einen (messbaren oder spürbaren) Unterschied zu machen, dann folgt, dass wir in solchen Fällen keine Pflichten haben. Dieses Problem wurde bereits von Derek Parfit erkannt und um es zu beheben, hat er argumentiert, dass nicht wahrnehmbare Auswirkungen unserer Handlungen moralisch signifikant sein können, wenn sie gemeinsam mit den Auswirkungen der Handlungen anderer, ähnlich positionierter Akteur*innen einen wahrnehmbaren positiven (oder negativen) Effekt haben (Parfit 1984). Sein Argument ist pragmatischer Natur:

"For the sake of small benefits to ourselves, or our families, each of us may deny others much greater total benefits, or impose on others much greater total harms. We may think this permissible because the effects on each of the others will be either trivial or im- 
perceptible. If this is what we think, what we do will often be much worse for all of us [...]. It is not enough to ask, 'Will my act harm other people? ‘ [...] I should ask, 'Will my act be one of a set of acts that will together harm other people? « (ebd., 86).

Anders gesagt, argumentiert Parfit im Wesentlichen, dass mitunter das kumulative Resultat eines Sets von Einzelhandlungen dafür ausschlaggebend ist, ob eine Einzelhandlung, die Teil des Sets ist, als moralisch richtig oder falsch anzusehen ist.

Widmen wir uns nun dem zweiten moraltheoretischen Problem, das durch den normativen Vorrang der kollektiven Ebene gelöst werden kann. Wie bereits erwähnt, was Probleme mit kollektiver Notwendigkeit betrifft, so kann jeweils kein ${ }^{*}$ Einzelakteur*in den Erfolg der erforderlichen Handlung garantieren; es gehören immer mindestens zwei dazu. Für Probleme mit strikter kollektiver Notwendigkeit gilt zudem dass der Erfolg der erforderlichen gemeinsamen Handlung von den Beitragshandlungen jedes/jeder Einzelnen abhängt (Schwenkenbecher 2017): die Anzahl der minimal erforderlichen Beitragenden ist gleich der Anzahl der verfügbaren Akteur*innen. Dies ist zum Beispiel im oben erwähnten Rettungsszenario von Garrett Cullity der Fall.

Allerdings ist es bei kollektiven Handlungsproblemen mit internationalen oder gar globalen Dimensionen normalerweise der Fall, dass es schlicht und ergreifend nicht jedes/jeder moralisch handlungsfähigen Akteurs/Akteurin bedarf, um diese Probleme zu lösen. Man kann die letztgenannte Eigenschaft solcher Probleme weite kollektive Notwendigkeit nennen (Schwenkenbecher 2017). Für kollektive Handlungsprobleme mit weiter kollektiver Notwendigkeit gilt, dass, obschon es mehrerer Einzelakteur ${ }^{\star}$ innen bedarf, solche Probleme zu lösen, die Anzahl der verfügbaren Akteur*innen zur Behebung des Problems größer ist als die Anzahl der minimal erforderlichen Akteur*innen und dass daher der kollektive Erfolg des gemeinsamen Handelns nicht kontrafaktisch von dem Beitrag jedes/jeder Einzelnen abhängt. Klarerweise handelt es sich bei weitreichenden kollektiven Handlungsproblemen wie Klimawandel oder globaler Armut nicht um Probleme mit strikter, sondern weiter kollektiver Notwendigkeit.

Wenn Einzelne nur dann Pflichten hätten, wenn diese kontrafaktische Abhängigkeit besteht, dann würde folgen, dass in Fällen mit weiter kollektiver Notwendigkeit keine Einzelperson eine Pflicht hat, sich eines kollektiven Handlungsproblems anzunehmen. Dass dies kontraintuitiv ist, wird am besten an einem Beispiel illustriert. Nehmen wir an, dass in Garrett Cullitys oben beschriebenem Rettungsfall statt der zwei Personen, die eine Winde bedienen müssen, um ei$n e^{\star} n$ Ertrinkende ${ }^{\star} n$ aus dem Wasser zu ziehen, vier Personen anwesend sind, und nur maximal zwei die Winde bedienen können. Die Weigerung eines Einzelnen, an der Rettungsaktion teilzunehmen, würde - für sich genommen - nicht dazu führen, dass der/die Ertrinkende nicht gerettet wird. Immerhin ist es nicht so, dass der Beitrag einer bestimmten Einzelperson notwendig ist, um die Aktion durchzuführen. Dies trifft jedoch auf jede $e^{\star}$ einzelne ${ }^{\star} n$ potentielle ${ }^{\star} n$ Akteur ${ }^{\star}$ in in dieser Situation zu. Keiner der vier anwesenden Akteur*innen ist streng genommen notwendig, um die Winde zu bedienen. Wenn nun die Hilfspflicht daran gekoppelt wäre, dass die jeweiligen Beitragshandlungen für die Rettung notwendig sind, dann ergäbe sich folgendes Problem, welches man das >Aufhebungsproblem`nennen kann: In dem Fall, wo vier potentielle Helfer*innen zur Verfügung stehen, hätte keiner der vier eine Pflicht, während in dem Fall wo nur zwei zur Verfügung stehen, diesen eine Hilfspflicht zukommt.

Es gibt zwei Möglichkeiten, dem Aufhebungsproblem zu begegnen. Entweder wird angenommen, dass im Falle der vier anwesenden Helfer ${ }^{*}$ innen jeweils nur ein beliebiges Zweierset von Akteur*innen die Hilfspflicht hat. Dies birgt jedoch eine weitere Schwierigkeit: jedwede Art und Weise, aus dem Viererset eine Zweierkombination auszuwählen und dieser eine Hilfspflicht zuzuschreiben muss notwendigerweise willkürlich sein (soweit es keine moralisch relevanten Unterschiede zwischen den vier Personen gibt). Zudem wäre es für die einzelnen Akteur*innen nicht ersichtlich, ob sie Teil des verpflichteten Zweiersets sind oder nicht. Die andere Möglichkeit, dem Aufhebungsproblem zu begegnen besteht darin, von einer kollektiven Hilfspflicht auszugehen, die alle vier potentiellen Helferinnen umfasst, wobei diese kollektive Pflicht noch unterbestimmt lässt, was genau jede ${ }^{\star}$ r Akteur ${ }^{\star}$ in $\mathrm{zu}$ tun hat und ob jede ${ }^{\star} \mathrm{r}$ Akteur ${ }^{\star}$ in handelnd aktiv werden muss. Der Vorschlag, dass die kollektive Ebene in diesem Sinne primär ist wurde u. a. von Bill Wringe und mir gemacht (Wringe 2016, Schwenkenbecher 2019).

Es lässt sich zusammenfassend sagen, dass Autor*innen mit dem Vorschlag, Verantwortung und moralische Pflichten als kollektiv zu verstehen, ausdrücken wollen, dass für unsere moralische Entscheidungsfindung sowie für die Bewertung unserer Handlungen oftmals die kollektive Ebene als Ausgangs- 
punkt dienen sollte: Was richtig oder falsch ist, wird mitunter dadurch bestimmt, was wir gemeinsam mit anderen erreichen bzw. anrichten können. Dies trifft vor allem auf Fälle zu, in denen wir allein nichts ausrichten können und nur gemeinsam mit anderen, ein erwünschtes Resultat erzielen können (kollektive Notwendigkeit), einschließlich jener Situationen, in denen die Effekte unserer Einzelhandlungen für sich genommen nicht messbar sind (individuelle Unwirksamkeit). Es betrifft weiterhin Fälle in denen es nicht jedes/jeder Handelnden bedarf, um ein gemeinsames Ziel zu erreichen (weite kollektive Notwendigkeit). Wenn wir unsere eigene Handlungspflicht von der kollektiven Notwendigkeit ableiten, dann wenden wir sogenanntes `we-reasoning an. Das heißt, wir schließen von einem Muster optimalen kollektiven Handelns auf die von uns erforderliche Beitragshandlung (vgl. Schwenkenbecher 2019).

Wenn die kollektive Ebene für die Entscheidungsfindung und die Handlungsbewertung in dem hier beschriebenen Sinn primär ist, dann kann von kollektiven Pflichten bzw. kollektiver Verantwortung im sschwachen Sinn gesprochen werden. Am Anfang dieses Abschnitts wurde erwähnt, dass Autor*innen, die einen kollektiven Verantwortungsbegriff vertreten, üblicherweise mindestens eine von zwei folgenden Strategien verfolgen. Hier nun die zweite Strategie:

2. Normative Verknüpfung von Akteur*innen: Der kollektive Charakter von Verantwortung und Pflichten kann des Weiteren so verstanden werden, dass man damit spezielle normative Verknüpfungen zwischen Akteur*innen, die gemeinsam eine Pflicht innehaben, deutlich machen möchte. Kollektive Verantwortung in diesem starken Sinne bedeutet, dass Gruppen von Akteur*innen jeweils Verantwortung für das Gelingen der gemeinsamen Handlung oder die Erzeugung des gemeinsamen Resultats mitverantwortlich sind. Dies umfasst Beitragshandlungen wie Handlungskoordination, die Verbesserung oder Produktion von gruppenbasiertem Wissen, welches Gruppenmitglieder benötigen, um eine Handlung auszuführen. Es kann außerdem bedeuten, dass Gruppenmitglieder in die Bresche springen müssen, wenn andere ihren Beitrag nicht leisten, selbst wenn sie dann mehr als ihren gerechten Anteil an der Gesamthandlung erbringen müssen. $\mathrm{Zu}$ guter Letzt, kann unter einer normativen Verknüpfung von Akteur*innen, die eine gemeinsame Pflicht haben, auch verstanden werden, dass diese anderen Gruppenmitgliedern gegenüber zu Fairness verpflichtet sind, wie zum Beispiel von Cullity argumentiert wurde (Cullity 2004; Schwenkenbecher 2020).

\subsection{Fazit}

Um nun abschließend zur Frage der kollektiven Verantwortung für (globale) Armut zurückzukommen: inwiefern lassen sich die oben gemachten Überlegungen auf globale Armut anwenden? Es lässt sich sagen, dass die meisten moralischen Akteur*innen vermutlich eine Vielzahl von individuellen und kollektiven Pflichten in Bezug auf Probleme wie (globale) Armut haben. Welche Pflichten wir genau haben, hängt von unserer jeweiligen Situation ab, vor allem davon, wo unsere Bemühungen einzeln oder gemeinsam den größten Effekt haben. Für viele von uns werden diese Pflichten nur im weiteren (und schwächeren) Sinne kollektiv sein, nämlich in dem Sinne, dass die kollektive Ebene für die Entscheidungsfindung und die Handlungsbewertung primär ist, wie unter (1) beschrieben (vgl. auch Schwenkenbecher 2019). Kollektivität der Verantwortung im stärkeren Sinn erfordert hingegen stärkere epistemische Verknüpfungen der Gruppenmitglieder sowie eine Bekenntnis zum gemeinsamen Ziel. Wo diese bereits bestehen, da können wir von kollektiver Verantwortung in diesem starken Sinn sprechen und entsprechend von den Mitgliedern dieser Gruppen einen größeren Einsatz für das Endresultat erwarten.

\section{Literatur}

Aas, Sean: Distributing Collective Obligation. In: Journal of Ethics and Social Philosophy 9/3 (2015), 1-23.

Aragon, Corwin/Jaggar, Alison M.: Agency, Complicity, and the Responsibility to Resist Structural Injustice. In: Journal of Social Philosophy 49/3 (2018), 439-460.

Ashford, Elizabeth: Severe Poverty as a Systemic Human Rights Violation. In: Gillian Brock (Hg.): Cosmopolitanism Versus Non-Cosmopolitanism. Critiques, Defenses, Reconceptualizations. Oxford 2013, 129-155.

Björnsson, Gunnar: Essentially Shared Obligations. In: Midwest Studies In Philosophy 38/1 (2014), 103-120.

Calder, Todd: Shared Responsibility, Global Structural Injustice, and Restitution. In: Social Theory and Practice 36/2 (2010), 263-290.

Collins, Stephanie: Collectives' Duties and Collectivisation Duties. In: Australasian Journal of Philosophy 91/2 (2013), 231-248.

Cullity, Garrett: The moral demands of affluence. Oxford 2004.

Lawford-Smith, Holly: The Feasibility of Collectives' Actions. In: Australasian Journal of Philosophy 90/3 (2012), 453-467.

Lichtenberg, Judith: Negative Duties, Positive Duties, and the >New Harms . In: Ethics 120/3 (2010), 557-578.

List, Christian/Pettit, Philip: Group agency. The possibility, design, and status of corporate agents. Oxford 2011. 
Murphy, Liam B.: Moral demands in nonideal theory. New York 2000.

Parfit, Derek: Reasons and Persons. Oxford 1984.

Pinkert, Felix: What We Together Can (Be Required to) Do. In: Midwest Studies In Philosophy 38/1 (2014), 187-202.

Pogge, Thomas W. M.: World poverty and human rights. Cosmopolitan responsibilities and reforms. Cambridge: 2002.

Schlothfeldt, Stephan: Individuelle oder gemeinsame Verpflichtung? Das Problem der Zuständigkeit bei der Behebung gravierender Übel. Paderborn 2009.

Schwenkenbecher, Anne: Joint Duties and Global Moral Obligations. In: Ratio 26/3 (2013), 310-328.

Schwenkenbecher, Anne: Gemeinsame Hilfspflichten, Weltarmut und kumulative Handlungen. In: Zeitschrift für Praktische Philosophie 4/1 (2017), 123-150.

Schwenkenbecher, Anne: Making sense of collective moral obligations: A comparison of current approaches. In: Tracy Isaacs/Kendy Hess/Violetta Igneski (Hg.): Collectivity: Ontology, Ethics and Social Justice. London 2018, 109-132.

Schwenkenbecher, Anne: Collective moral obligations: ‘we- reasoning and the perspective of the deliberating agent. In: The Monist 102/2 (2019), 151-171.

Schwenkenbecher, Anne: Structural injustice and massively shared obligations. In: Journal of Applied Philosophy 1-16 (2020). DOI: 10.1111/japp.12431.

Wringe, Bill: Needs, Rights, and Collective Obligations. In: Royal Institute of Philosophy Supplement 80/57 (2005), 187-207.

Wringe, Bill: Global obligations and the agency objection. In: Ratio 23/2 (2010), 217-231.

Wringe, Bill: From Global Collective Obligations to Institutional Obligations. In: Midwest Studies In Philosophy 38/1 (2014), 171-186.

Wringe, Bill: Collective Obligations. Their Existence, Their Explanatory Power, and Their Supervenience on the Obligations of Individuals. In: European Journal of Philosophy 24/2 (2016), 472-497.

Young, Iris Marion: Responsibility and Global Labor Justice. In: Journal of Political Philosophy 12/4 (2004), 365-388.

Young, Iris Marion: Responsibility for justice. New York 2011.

Anne Schwenkenbecher 